\title{
Spatial impulse response of a rectangular double curved transducer
}

Bæk, David; Jensen, Jørgen Arendt; Willatzen, Morten

Published in:

Journal of the Acoustical Society of America

Link to article, DOI:

$10.1121 / 1.3693659$

Publication date:

2012

Document Version

Early version, also known as pre-print

Link back to DTU Orbit

Citation (APA):

Bæk, D., Jensen, J. A., \& Willatzen, M. (2012). Spatial impulse response of a rectangular double curved transducer. Journal of the Acoustical Society of America, 131(4), 2730-2741. https://doi.org/10.1121/1.3693659

\section{General rights}

Copyright and moral rights for the publications made accessible in the public portal are retained by the authors and/or other copyright owners and it is a condition of accessing publications that users recognise and abide by the legal requirements associated with these rights.

- Users may download and print one copy of any publication from the public portal for the purpose of private study or research.

- You may not further distribute the material or use it for any profit-making activity or commercial gain

- You may freely distribute the URL identifying the publication in the public portal

If you believe that this document breaches copyright please contact us providing details, and we will remove access to the work immediately and investigate your claim. 
Spatial impulse response of a rectangular double curved transducer

David Bæk ${ }^{\mathrm{a})}$ and Jørgen Arendt Jensen

Center for Fast Ultrasound Imaging,

Department of Electrical Engineering,

Technical University of Denmark,

Ørsteds Plads Building 349,

2800 Kgs. Lyngby,

Denmark

Morten Willatzen

Mads Clausen Institute for Product Innovation,

University of Southern Denmark,

Alsion,

6400 Sønderborg,

Denmark

(Dated: January 11, 2012)

Convex elevation focused transducers 


\begin{abstract}
Calculation of the pressure field from transducers having both a convex and a concave surface geometry is a complicated assignment that often is accomplished by subdividing the transducer surface into smaller flat elements of which the spatial impulse response is known. This method is often seen applied to curved transducers because an analytical solution is un-known. In this work a semi-analytical algorithm for the exact solution to a first order in diffraction effect of the spatial impulse response of rectangular shaped double curved transducers is presented. The algorithm and an approximation of it are investigated. The approximation reformulates the algorithm to an analytically integrable expression which is computationally efficient to solve. Simulation results are compared with the simulation software Field II. Calculating the response from 200 different points yields a mean error for the different approximations ranging from $0.03 \%$ to $0.8 \%$ relative to a numerical solution for the spatial impulse response. It is shown that the presented algorithm gives consistent results with Field II for a linear flat, a linear focused, and a convex non-focused element. Best solution was found to be $0.01 \%$ with a three-point Taylor expansion.
\end{abstract}

PACS numbers: 43.38.Hz,43.20.Px,43.40.Rj 


\section{INTRODUCTION}

Calculation of the spatial impulse responses (SIR) for predicting acoustic pressure and pulse-echo responses has been a well known technique for decades. Some of the first works utilizing SIRs on planar transducers were by Tupholme and Stepanishen ${ }^{1,2}$ and later several analytical expressions for rectangular, circular, concave circular, and array transducer have been reported ${ }^{3-11}$. Expressions for transducers with slightly in-homogenous surface movement have also been introduced ${ }^{12,13}$. However, most of the reported solutions represent simple transducer geometries that are rarely found in more sophisticated acoustic applications, e.g. medical imaging applications. The simple planar solutions may be utilized to calculate SIRs of the more complicated surface geometries such as annular arrays, linear elevation focused transducers, convex transducers, and double curved transducers, i.e. rectangular transducers with a convex geometry and an elevation focused geometry. To achieve this a subdivision of the transducer surface into smaller simpler elements such as triangles or rectangles is often performed. The final response is then calculated by applying superposition of the responses from many smaller planar elements. An example where this principle is practically applied is the Field II simulation software package $\mathrm{e}^{14,15}$. This package utilizes the algorithms described by Jensen ${ }^{8,16,17}$.

Only a minor part of the literature within SIRs addresses the problem of finding analytical expressions of curved rectangular transducers. Within this literature the work ${ }^{18-20}$ shows that subdivision of the elements into small stripes or rectangles is possible. The motivation for applying these assumptions is that no analytical solution has been found for these relatively complicated geometries. Theumann et al. ${ }^{21}$ formulated a semi-analytical expression for points inside a closed cylinder. To model the response from slightly curved transducer elements, which often are found in medical imaging, simulation tools such as Ultrasim ${ }^{22}$, DREAM $^{23}$, DELFI ${ }^{24}$, and Field II typically apply a discretization of the surface into smaller elements as mentioned above whereby they efficiently can solve the Rayleigh integral with

\footnotetext{
a) Electronic address: db.mechatronic@gmail.com
} 
its planar assumptions.

Alternative solutions to the problem are finite element implementations. A finite element method implementation can account for second order diffraction effects due to surface curvature, shear waves and attenuation. Finite element methods can be made almost arbitrarily detailed but numerical problems can occur in the implementation of open boundary conditions for limiting the fluid domain and in the resolution of e.g. a tissue domain to account for the complicated acoustic scattering. Also finite element solutions are inherently slow, which significantly limits their use in studying complex multi-element transducers used for advanced imaging like 3D and flow. There is, thus, a need for analytic and faster solutions.

This paper presents an exact expression for the SIR of a rectangular convex elevation focused transducer (or double curved transducer) in the form of an elliptical integral. This integral is shown to be solvable by applying either a Taylor expansion or fitting a second order polynomial to a part of the elliptical integral. The motivation for developing such algorithms is connected with an attempt to make the Field II software more efficient in solving the response from double curved surfaces. Double curved surfaces are present in the medical ultrasound industry of today but an efficient solver for multiple array elements, as can easily be found for linear areas, is not present. A research on finding an efficient alternative to subdividing the double curved surface into smaller elements has therefore been of high interest and may potentially speed up the solving process of such transducer geometries.

\section{THEORY}

The general mathematical formulation of the SIR can be extracted from the Rayleigh integral $^{1,2}$ to yield:

$$
h(\vec{r}, t)=\frac{1}{2 \pi} \int_{S} \frac{\delta\left(t-\frac{|\vec{r}|}{c}\right)}{|\vec{r}|} d S
$$

The validity of this equation is subject to the assumption that the wavelength is much smaller than the curvature of the transducer so that the secondary diffraction effects are 


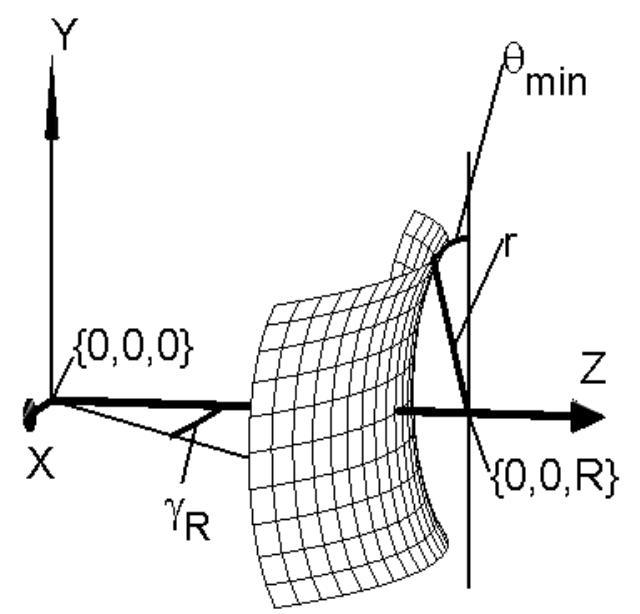

FIG. 1. The geometrical definition of the double curved transducer.

negligible ${ }^{10,25}$. It is furthermore assumed that the surface movement is uniform and that a piston movement is forced onto the surface. The transducer surface transmits into a fluid or tissue in a longitudinal single mode operation of the solid.

A torus can be formed by an outer revolution angle $\gamma$ and an inner revolution angle $\theta$ describing a circle that rides on the outer circle. The angles are limited within $0-2 \pi$ for $\gamma$ and $0-\pi$ for $\theta$.

A double curved transducer as considered in this work is defined by the torus coordinates

$$
\begin{aligned}
& x=(R-r \sin \theta) \sin \gamma \\
& z=(R-r \sin \theta) \cos \gamma, \\
& y=r \cos \theta,
\end{aligned}
$$

where the angles $\gamma$ and $\theta$ are the revolving angles and $R$ and $r$ define the outer and the inner circle radii, respectively. Figure 1 depicts the boundary limiting angles and the geometry. The maximum and minimum opening angles for the concave curvature are defined as $\theta_{\min }$ and $\theta_{\max }$, where $\theta_{\max }=\pi-\theta_{\min }$. Similarly do $\gamma_{L}$ and $\gamma_{R}$ define the limiting angles of the convex curvature and due to the transducer symmetry $\gamma_{R}=-\gamma_{L}$ is valid. It should be noted that in this work the positive angle definition for $\gamma$ is calculated CCW around the y-axis and 


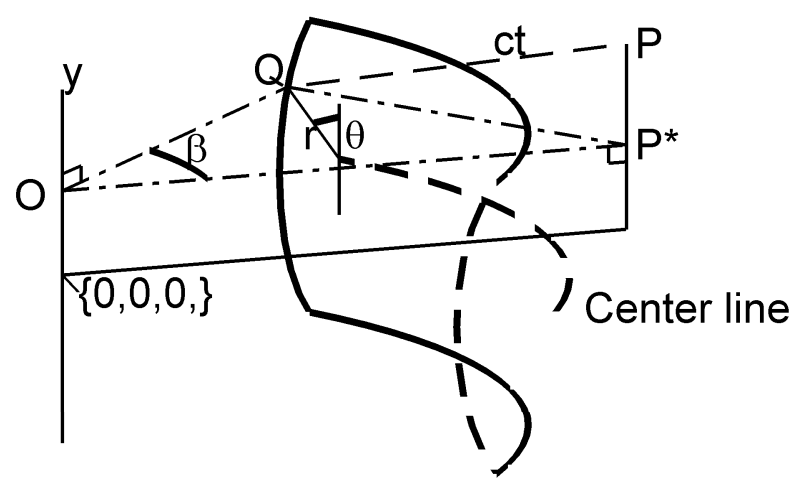

FIG. 2. Figure showing the geometrical definition of the angle $\beta$.

relative to the z-axis. The angle $\gamma_{R}$ is therefore negative. Furthermore, the $\theta$ angle definition has its zero reference from the vertical line going through the point $\{0,0, R\}$ parallel to the y-axis, hence $0 \leq \theta \leq \pi$. All Cartesian coordinates are relative to the $\{0,0,0\}$ coordinate. A point, $P=\left\{x_{p}, y_{p}, z_{p}\right\}$, can be placed at any location in front of the transducer, to the left and to the right, and below or above the transducer. The only requirement for the point's location is that a spherical wave emitted from the location does not meet the back of the concave transducer before meeting the front.

To perform the integration in (1) a definition of the surface element, $d S$, on the torus surface $S$ is needed

$$
d S=r(R-r \sin \theta) d \gamma d \theta
$$

which is valid when the torus is parametrically defined as $T(\gamma, \theta)=z \vec{i}+x \vec{j}+y \vec{k}$.

By considering Fig. 2 and by applying cosine relations one can obtain an expression for the angle $\beta$ as

$$
\begin{aligned}
\beta & =\cos ^{-1}\left(\frac{\left|O P^{*}\right|^{2}+(R-r \sin \theta)^{2}-\left(c^{2} t^{2}-\left(y_{p}-r \cos \theta\right)^{2}\right)}{2(R-r \sin \theta)\left|O P^{*}\right|}\right) \\
& =\cos ^{-1}\left(\frac{k-c^{2} t^{2}-2 r y_{p} \cos \theta-2 r R \sin \theta}{2\left|O P^{*}\right| R-2\left|O P^{*}\right| r \sin \theta}\right),
\end{aligned}
$$


where $t$ is the time and $c$ is the speed of sound and $k=\left|O P^{*}\right|^{2}+r^{2}+R^{2}+y_{p}^{2}$. The angle $\beta$ is defined to rotate around the y-axis as shown in Fig. 2.

At any time instant the surface element, $d S$, can be found as

$$
\begin{aligned}
d S & =r(R-r \sin \theta) \frac{\partial \beta}{\partial t} d t d \theta \\
& =r(R-r \sin \theta) \frac{2 c^{2} t}{\left(2\left|O P^{*}\right| R-2\left|O P^{*}\right| r \sin \theta\right) \sqrt{1-\frac{\left(-k+c^{2} t^{2}+2 r y_{p} \cos \theta+2 r R \sin \theta\right)^{2}}{\left(2\left|O P^{*}\right| R-2\left|O P^{*}\right| r \sin \theta\right)^{2}}}} d t d \theta .
\end{aligned}
$$

By substituting (7) into (1) the integral for the SIR becomes:

$$
h\left(t_{i}\right)=\frac{1}{2 \pi} \int_{\theta_{\min }\left(t_{i}\right)}^{\theta_{\max }\left(t_{i}\right)} \frac{c r}{\left|O P^{*}\right| \sqrt{1-\frac{\left(-k+c^{2} t^{2}+2 r y_{p} \cos \theta+2 r R \sin \theta\right)^{2}}{\left(2\left|O P^{*}\right| R-2\left|O P^{*}\right| r \sin \theta\right)^{2}}}} d \theta .
$$

The surface integral in (1) has hereby been transformed into a line integral of elliptical form that integrates along the intersection between a crossing sphere and the transducer.

The integration boundaries $\theta_{\min }$ and $\theta_{\max }$ are to be found from (6). A general expression for the angle $\theta$ is found by isolating it in (6) for a given angle of $\beta$. This yields four solutions of which two are valid in the integration domain defined for this type of transducer. The two remaining angles are to be used if $\pi \leq \theta \leq 2 \pi$.

$$
\theta=\cos ^{-1}\left(\frac{f_{1} \pm f_{2}}{f_{3}}\right)
$$

where

$$
\begin{aligned}
f_{1} & \left.=2 r y_{p}\left(k-c^{2} t^{2}-2\left|O P^{*}\right| R \cos (\beta)\right)\right), \\
f_{2} & =\left(( 2 r R - 2 | O P ^ { * } | r \operatorname { c o s } ( \beta ) ) ^ { 2 } \left(-\left(k-c^{2} t^{2}\right)^{2}+(2 r)^{2}\left(R^{2}+y_{p}^{2}\right)+\ldots\right.\right. \\
& \left.\left.+\left(2\left|O P^{*}\right| \cos (\beta)\left(2 R\left(k-2 r^{2}-c^{2} t^{2}\right)+2\left|O P^{*}\right|\left(r^{2}-R^{2}\right) \cos (\beta)\right)\right)\right)\right)^{1 / 2}, \\
f_{3} & =4 r^{2}\left(R^{2}+y_{p}^{2}+\left|O P^{*}\right| \cos (\beta)\left(\left|O P^{*}\right| \cos (\beta)-2 R\right)\right) .
\end{aligned}
$$

There are three values of $\beta$ to which the integration boundaries correspond. These are for the angles at which the intersecting curve crosses the vertical tranducer edges, and for the angle at which the intersecting closed curve can be split into two arcs by a vertical plane through $\mathrm{P}$ and the $\mathrm{y}$-axis. The values are $\beta_{l}=\left(\gamma_{L}-\gamma_{p}\right), \beta_{r}=\left(\gamma_{R}-\gamma_{p}\right)$, and $\beta_{0}=0$, where

$$
\gamma_{p}=\tan ^{-1}\left(\frac{x_{p}}{z_{p}}\right)
$$




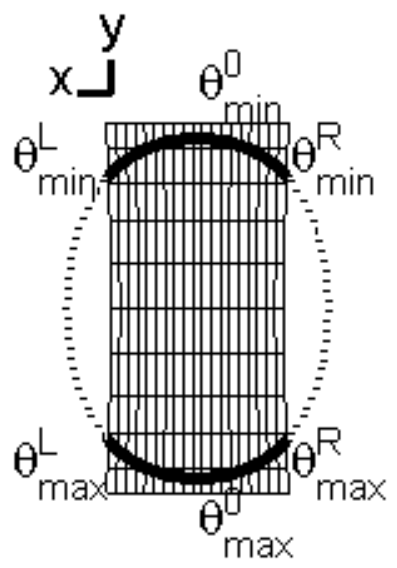

FIG. 3. The definition of the integration angles $\theta_{\min }^{L}, \theta_{\max }^{L}, \theta_{\min }^{R}$, and $\theta_{\max }^{R}$, which occur when the spherical wave has passed the boundaries of the transducer. The transducer is seen from the back side toward the positive z-axis.

The angle $\gamma_{p}$ is the angle at which the point $\mathrm{P}$ is located relative to the $\mathrm{z}$-axis in the xz-plane.

Fig. 3 illustrates a transducer seen from the back side in the xy-plane and towards the positive z-axis. The solid arcs are defining the intersection between a sphere emanating from a point $\mathrm{P}$ and the transducer. The dotted lines are the sphere's crossing with a imaginary extension of the transducer. The boundary integration angles $\theta_{\text {min }}^{R}, \theta_{\text {max }}^{R}, \theta_{\text {min }}^{L}, \theta_{\text {max }}^{L}, \theta_{\text {min }}^{0}$, and $\theta_{\text {max }}^{0}$ are dependent on $\beta$ and time as defined in (9) and they split the closed arc into the sub arcs illustrated. E.g. the angles $\theta_{\min }^{0}$ and $\theta_{\max }^{0}$ "split" the solid arcs into a left and a right arc line. For the depicted situation in Fig. 3 the SIR found from (8) is calculated by organizing the integration angles as

$$
h=2 \frac{1}{2 \pi} \int_{\theta_{\min }^{0}}^{\theta_{\max }^{0}} I(\theta) d \theta-\frac{1}{2 \pi} \int_{\theta_{\min }^{L}}^{\theta_{\max }^{L}} I(\theta) d \theta-\frac{1}{2 \pi} \int_{\theta_{\min }^{R}}^{\theta_{\max }^{R}} I(\theta) d \theta
$$

where $I(\theta)$ is the integrand found in (8). 
It is beneficial to define two functions for the integration angles as:

$$
\begin{gathered}
\theta\left(t, \chi, t_{s}, \theta_{s}, t_{e}, \theta_{e}, \beta\right)= \begin{cases}\theta_{s} & t \leq t_{s} \\
\cos ^{-1}\left(\frac{f_{1}(\beta, t)+\chi f_{2}(\beta, t)}{f_{3}(\beta, t)}\right) & t_{s}<t<t_{e} \\
\theta_{e} & t \geq t_{e},\end{cases} \\
\left.\theta_{\text {single }}(t, \chi, \beta)\right)=\cos ^{-1}\left(\frac{f_{1}(\beta, t)+\chi f_{2}(\beta, t)}{f_{3}(\beta, t)}\right) .
\end{gathered}
$$

Here $t_{s}$ defines a lower time at which the integration angle is a constant. Similarly, $t_{e}$ defines an upper time at which the angle is constant. In between these times the angle $\theta$ takes on a time dependent value. The constant $\chi$ takes on the values 1 or -1 and determines the sign in front of the function $f_{2}$. The function $\theta_{\text {single }}$ is useful for calculating single angle values, which is necessary in some of the follow definitions of the SIR. By defining the integration angles as having a value at all time instants the number of time branching needed to define the SIR is limited. It is hereby meant that the pulse will have a starting time which corresponds to the time at which the sphere touches the aperture for the first time. Similarly, the pulse has an end time. In between these two times there are many intersections between the edges of the aperture and the sphere present. Therefore, by properly subtracting and adding line contributions as shown in (14) each time the sphere crosses a boundary it becomes possible to account for all possible point locations relative to the transducer's surface.

\section{TIME OF FLIGHT DEFINITIONS}

To fully describe all possible locations of a point in front of the transducer one needs to define nine time of flight values. These times are given by the distances from the point, $\mathrm{P}$, to each transducer corner, the shortest distances to the side edges at $\gamma_{L}$ and $\gamma_{R}$, the shortest distances to the upper and the lower horizontal edges at $\theta_{\max }$ and $\theta_{\min }$, and the shortest distances to the transducer. The transducer corners are defined as $c_{1}, c_{2}, c_{3}$, and $c_{4}$. Corner 
coordinates are defined as $c_{i}=\{x, y, z\}$ :

$$
\begin{aligned}
& c_{1}=\left\{\left(R-r \sin \left(\theta_{\text {min }}\right)\right) \sin \left(\gamma_{L}\right), r \cos \left(\theta_{\text {min }}\right),\left(R-r \sin \left(\theta_{\text {min }}\right)\right) \cos \left(\gamma_{L}\right)\right\}, \\
& c_{2}=\left\{\left(R-r \sin \left(\theta_{\text {max }}\right)\right) \sin \left(\gamma_{L}\right), r \cos \left(\theta_{\text {max }}\right),\left(R-r \sin \left(\theta_{\text {max }}\right)\right) \cos \left(\gamma_{L}\right)\right\}, \\
& c_{3}=\left\{\left(R-r \sin \left(\theta_{\text {min }}\right)\right) \sin \left(\gamma_{R}\right), r \cos \left(\theta_{\min }\right),\left(R-r \sin \left(\theta_{\min }\right)\right) \cos \left(\gamma_{R}\right)\right\}, \\
& c_{4}=\left\{\left(R-r \sin \left(\theta_{\text {max }}\right)\right) \sin \left(\gamma_{R}\right), r \cos \left(\theta_{\text {max }}\right),\left(R-r \sin \left(\theta_{\text {max }}\right)\right) \cos \left(\gamma_{R}\right)\right\} .
\end{aligned}
$$

Coordinates for the shortest distances to the vertical edges at $\gamma_{L}$ and $\gamma_{R}$ are geometrically differently defined according to the location of the point, $\mathrm{P}$, and in this work are referred to as $c_{L}$, and $c_{R}$, respectively. Similar situations occur for coordinates defining the shortest distance to the upper and the lower horizontal transducer edges. The locations of these horizontal edges are defined by $\theta_{\min }$ as shown in Fig. 1 and $\theta_{\max }=\pi-\theta_{\min }$. The coordinates to the shortest distances at these locations are defined as $c_{5}$ and $c_{6}$ for the edges associated with $\theta_{\min }$ and $\theta_{\max }$, respectively. The location of the coordinate for the shortest distance to the transducer is denoted $c_{0}$.

The time of flights associated with the different coordinates are hereafter defined as $t_{c_{0}}$, $t_{c_{1}}, t_{c_{2}}, t_{c_{3}}, t_{c_{4}}, t_{c_{5}}, t_{c_{6}}, t_{c_{L}}$, and $t_{c_{R}}$.

\section{THE DIFFERENT ZONES}

The final integral expression in (1) takes on different forms depending on the location of the point, $\mathrm{P}$, relative to the transducer. In this work there are ten zones in front of the transducer defined. Points located in these zones share the zone specific integral form. 
Zone 1: $\theta_{\min } \leq \theta_{p} \leq \theta_{\max },\left|O P^{*}\right|<R, \gamma_{R} \leq \gamma_{p} \leq \gamma_{L}$,

Zone 2: $\theta_{\min } \geq \theta_{p} \leq \theta_{\max },\left|O P^{*}\right|<R, \gamma_{L} \leq \gamma_{p} \leq \gamma_{R}$,

Zone 3: $\left(\left|\theta_{p}\right|>\theta_{\max }||\left|\theta_{p}\right|<\theta_{\min }\right), \gamma_{R} \leq \gamma_{p} \leq \gamma_{L}, y_{p} \neq 0,\left|O P^{*}\right| \geq R$,

Zone 4: $\left(\left(\gamma_{p}<\gamma_{R}\right) \|\left(\gamma_{p}>\gamma_{L}\right)\right),\left|O P^{*}\right|>R, \theta_{\min }<\left|\theta_{p}\right|<\theta_{\max }$,

Zone 5: $\theta_{\text {min }}<\left|\theta_{p}\right|<\theta_{\max }, \gamma_{L} \geq \gamma_{p} \geq \gamma_{R},\left|O P^{*}\right|>R$,

Zone 6: $\left(\left(\theta_{\max }<\theta_{p}\right) \|\left(\theta_{\min }>\theta_{p}\right)\right),\left(\left(\gamma_{p}<\gamma_{R}\right) \|\left(\gamma_{p}>\gamma_{L}\right)\right), y_{p} \neq 0,\left|O P^{*}\right|<R$,

Zone \%: $\left(\left(\left|\theta_{p}\right|>\theta_{\max }\right)||\left(\left|\theta_{p}\right|<\theta_{\min }\right)\right), \gamma_{R} \leq \gamma_{p} \leq \gamma_{L}, y_{p} \neq 0,\left|O P^{*}\right|<R$,

Zone 8: $\left(\left(\theta_{\max }<\left|\theta_{p}\right|\right) \|\left(\theta_{\min }>\left|\theta_{p}\right|\right)\right),\left(\left(\gamma_{p}<\gamma_{R}\right) \|\left(\gamma_{p}>\gamma_{L}\right)\right), y_{p} \neq 0,\left|O P^{*}\right| \geq R$,

Zone 9: $y_{p}=0,\left|O P^{*}\right|=R$,

Zone 10: $\left|O P^{*}\right|=R, \gamma_{L} \leq \gamma_{p} \leq \gamma_{R}, y_{p}=0$.

See ZoneVideo. [Link to zoneVideo.avi]

\section{A. Spatial impulse response for Zone 1}

This zone is located directly in front of the transducer and in front of the elevation focus. A sphere emanating from within this zone may intersect the transducer as shown in Fig. 4a and Fig. 4b. Initially, the crossing will be a closed trajectory with upper and lower integration boundaries $\theta_{\min }^{0}$ and $\theta_{\max }^{0}$ as seen in Fig. 4a. The angle $\theta_{\min }^{0}$ gets equal to $\theta_{\min }$ when the crossing exceeds the upper horizontal edge of the transducer. A similar situation occurs for the $\theta_{\max }^{0}$ that equals $\theta_{\max }$ when the crossing exceeds the lower horizontal edge of the transducer. For the time instants, at which the intersections have not yet exceeded the vertical side of the transducer, the SIR is calculated as

$$
h=2 \frac{1}{2 \pi} \int_{\theta_{\min }^{0}}^{\theta_{\max }^{0}} I(\theta) d \theta,
$$

where $I(\theta)$ is the integrand found in (8). The factor of two is used because the integration only integrates along one of the two line segments and one has to integrate along the left and the right trajectory.

At the time instants at which the intersections crosses the vertical sides of the transducer, i.e $\gamma_{R}$ and $\gamma_{L}$, two new sets of integration limits occur: $\theta_{\max }^{R}$ and $\theta_{\min }^{R}$ for $\gamma_{R}$ and also $\theta_{\max }^{L}$ 

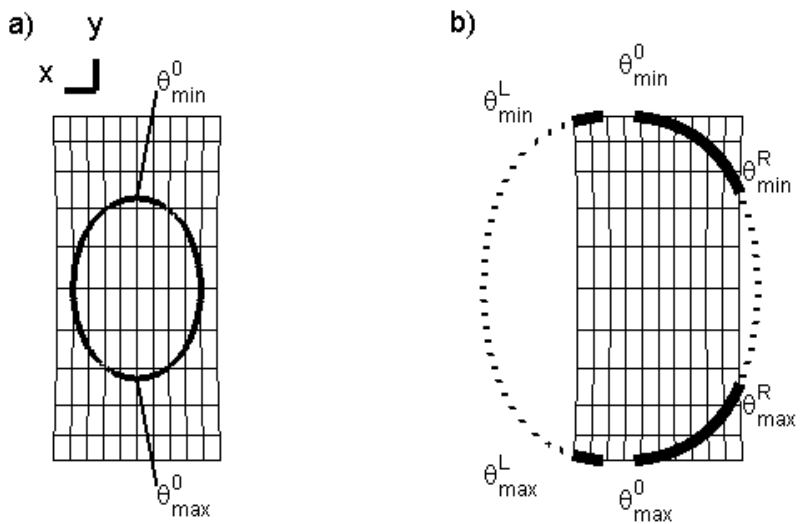

FIG. 4. A sphere's crossing with a transducer (solid) and virtual crossings (dotted). View seen from the transducer's back side in direction of the z-axis. a) The sphere has not yet crossed the side edges. The sphere is symmetrically placed at $x_{p}=0$. b) The sphere has crossed the edges. Dotted lines indicate the virtual arc crossings. The sphere center is offset to the left, which makes the left virtual arc significantly longer than the right virtual arc. See AngleVideo. Link to AngleVideo.avi

and $\theta_{\min }^{L}$ for $\gamma_{L}$ as seen in Fig. 4b. The SIR can then be calculated following one of two principles. The first principle relies on integrating along arcs that actually intersect the transducer (See solid lines on Fig. 4). The SIR is then formulated as

$$
h=\frac{1}{2 \pi} \int_{\theta_{\min }^{0}}^{\theta_{\min }^{R}} I(\theta) d \theta+\frac{1}{2 \pi} \int_{\theta_{\min }^{0}}^{\theta_{\min }^{L}} I(\theta) d \theta+\frac{1}{2 \pi} \int_{\theta_{\max }^{R}}^{\theta_{\max }^{0}} I(\theta) d \theta+\frac{1}{2 \pi} \int_{\theta_{\max }^{L}}^{\theta_{\max }^{0}} I(\theta) d \theta .
$$

The second method relies on calculating contributions from arc lengths located outside the transducer geometry as if the transducer was violating the limitations by $\gamma_{L} \leq \gamma \leq \gamma_{R}$. One should then subtract contributions from these virtual arcs from the total response. A mathematical formulation of this can be found as

$$
h=2 \frac{1}{2 \pi} \int_{\theta_{\min }^{0}}^{\theta_{\max }^{0}} I(\theta) d \theta-\frac{1}{2 \pi} \int_{\theta_{\min }^{L}}^{\theta_{\max }^{L}} I(\theta) d \theta-\frac{1}{2 \pi} \int_{\theta_{\min }^{R}}^{\theta_{\max }^{R}} I(\theta) d \theta .
$$

The latter principle may be considered more stable than the first principle because it has fewer small arc contributions. Throughout this work the second principle is utilized for 
formulating the SIR in all zones. Note, however, that the first integration principle will give exactly the same result and could just as well have been used.

The complete SIR integral for the zone in focus is:

$$
\begin{aligned}
h_{\text {zone } 1}(t)= & 2 h\left(\theta\left(t, 1, t_{0}, \theta_{0}, t_{c_{5}}, \theta_{\text {min }}, \beta_{0}\right), \theta\left(t,-1, t_{0}, \theta_{0}, t_{c_{6}}, \theta_{\text {max }}, \beta_{0}\right)\right)- \\
& h\left(\theta\left(t, 1, t_{0}, \theta_{R}, t_{c_{3}}, \theta_{\text {min }}, \beta_{r}\right), \theta\left(t,-1, t_{0}, \theta_{R}, t_{c_{4}}, \theta_{\text {max }}, \beta_{r}\right)\right)- \\
& h\left(\theta\left(t, 1, t_{0}, \theta_{L}, t_{c_{1}}, \theta_{\text {min }}, \beta_{l}\right), \theta\left(t,-1, t_{0}, \theta_{L}, t_{c_{2}}, \theta_{\text {max }}, \beta_{l}\right)\right) .
\end{aligned}
$$

This pulse is therefore defined from the minimum time instant, $t_{0}$, to the maximum time instant, which is one of the times $t_{c 1}, t_{c 2}, t_{c 3}, t_{c 4}, t_{c 5}$, or $t_{c 6}$. Note that (24) accounts for the different edge times and angle limitations through the formulation of the analytical function $\theta$ in $(15)$.

A short analysis of the integration angles represented in (24) can be performed for a given case by plotting the different $\theta$ angles as shown in Fig. 5. The considered situation is shown for a point located at $\left\{x_{p}, y_{p}, z_{p}\right\}=\{5,0,45\} \mathrm{mm}$ near a transducer with a height of $30 \mathrm{~mm}$, outer radius, $\mathrm{R}$, of $60 \mathrm{~mm}$, inner radius, $\mathrm{r}$, of $90 \mathrm{~mm}$, and a $\gamma_{L}=0.26$. The point is therefore placed in the xz-plane wherefore $t_{c_{5}}=t_{c_{6}}$. Furthermore, the point is placed to the left $\left(x_{p}>0\right)$ of the z-axis, which results in $t_{c_{L}}<t_{c_{R}}$ and $t_{c_{1}}=t_{c_{2}}<t_{c_{3}}=t_{c_{4}}$. The contribution from the virtual arc to the left of the transducer is therefore only nonzero in the time interval $t_{c_{L}} \leq t \leq t_{c_{3}}$, and for the right virtual arc it is the time interval $t_{c_{R}} \leq t \leq t_{c_{3}}$. Notice also that (15) introduces a cut off at $t_{c_{1}}$ and $t_{c_{2}}$, which are the time instants at which the virtual arc length to the left of the transducer exceeds the minimum and maximum opening angles $\theta_{\min }$ and $\theta_{\max }$.

\section{B. Spatial impulse response for Zone 2 to Zone 10}

The same analysis principle as applied for Zone 1 can be applied for all other zones. 


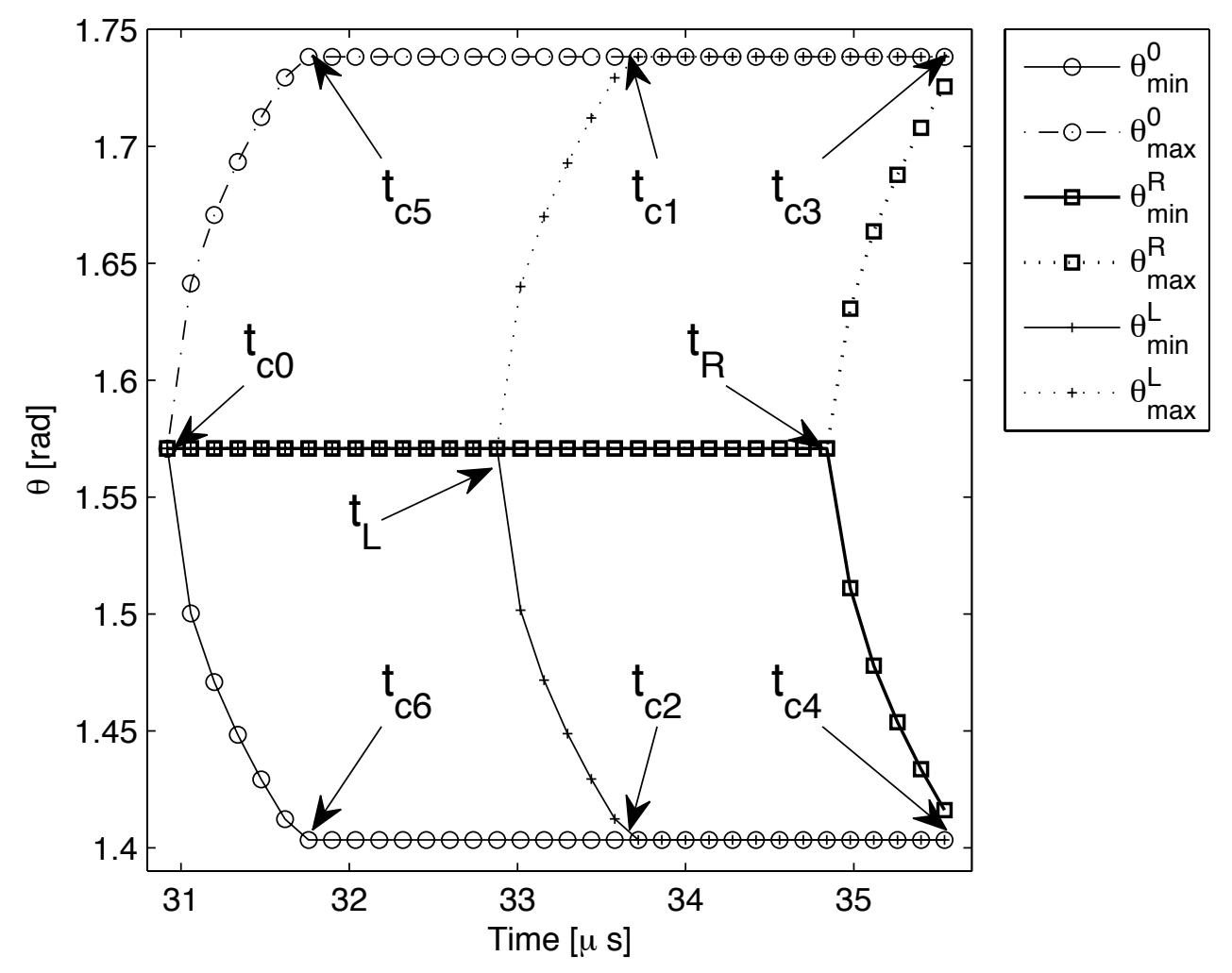

FIG. 5. The integration angles plotted for a point located in Zone 1 of a transducer. The angles show that the wave crosses the left edge before it crosses the right. Start and end times are $t_{c_{0}}$ and $t_{c_{3}}$, respectively.

\section{Zone 2}

For Zone 2 the SIR formulation is dependent on the point's location being to the right or to the left of the transducer. The responses are formulated as

$$
\begin{aligned}
& \text { For } \gamma_{p}<\gamma_{R} \\
& \begin{aligned}
h_{z o n e 2}(t)= & h\left(\theta\left(t, 1, t_{c_{R}}, \theta_{c_{R}}, t_{c_{3}}, \theta_{\text {min }}, \beta_{r}\right), \theta\left(t,-1, t_{c_{R}}, \theta_{c_{R}}, t_{c_{4}}, \theta_{\text {max }}, \beta_{r}\right)\right)- \\
& h\left(\theta\left(t, 1, t_{c_{L}}, \theta_{c_{L}}, t_{c_{1}}, \theta_{\text {min }}, \beta_{l}\right), \theta\left(t,-1, t_{c_{L}}, \theta_{c_{L}}, t_{c_{2}}, \theta_{\text {max }}, \beta_{l}\right)\right) .
\end{aligned}
\end{aligned}
$$

For $\gamma_{p}>\gamma_{L}$

$$
\begin{aligned}
h_{\text {zone } 2}(t)= & h\left(\theta\left(t, 1, t_{c_{L}}, \theta_{c_{L}}, t_{c_{1}}, \theta_{\text {min }}, \beta_{l}\right), \theta\left(t,-1, t_{c_{L}}, \theta_{c_{L}}, t_{c_{2}}, \theta_{\text {max }}, \beta_{l}\right)\right)- \\
& h\left(\theta\left(t, 1, t_{c_{R}}, \theta_{c_{R}}, t_{c_{3}}, \theta_{\text {min }}, \beta_{r}\right), \theta\left(t,-1, t_{c_{R}}, \theta_{c_{R}}, t_{c_{4}}, \theta_{\max }, \beta_{r}\right)\right) .
\end{aligned}
$$




\section{Zone 3}

For Zone 3 the SIR is formulated as

If $y_{p}>0$

$$
\begin{aligned}
& \chi_{r}= \begin{cases}1, & \theta_{\text {single }}\left(t_{c_{3}}, 1, \beta_{r}\right)=\theta_{\text {min }} \\
-1, & \text { else }\end{cases} \\
& \chi_{l}= \begin{cases}1, & \theta_{\text {single }}\left(t_{c_{1}}, 1, \beta_{l}\right)=\theta_{\text {min }} \\
-1, & \text { else }\end{cases}
\end{aligned}
$$

$$
\begin{aligned}
h_{\text {zone3 } 3}(t)= & 2 h\left(\theta\left(t, 1, t_{c_{5}}, \theta_{\text {min }}, t_{c_{5}}, \theta_{\text {min }}, \beta_{o}\right), \theta\left(t, 1, t_{c_{5}}, \theta_{\text {min }}, t_{c_{6}}, \theta_{\text {max }}, \beta_{o}\right)\right)+ \\
& h\left(\theta\left(t, \chi_{r}, t_{c_{3}}, \theta_{\text {min }}, t_{c_{3}}, \theta_{\text {min }}, \beta_{r}\right), \theta\left(t, \chi_{r}, t_{c_{3}}, \theta_{\text {min }}, t_{c_{4}}, \theta_{\text {max }}, \beta_{r}\right)\right)- \\
& h\left(\theta\left(t, \chi_{l}, t_{c_{1}}, \theta_{\text {min }}, t_{c_{1}}, \theta_{\text {min }}, \beta_{l}\right), \theta\left(t, \chi_{l}, t_{c_{1}}, \theta_{\text {min }}, t_{c_{2}}, \theta_{\text {max }}, \beta_{l}\right)\right),
\end{aligned}
$$

and for $y_{p}<0$

$$
\begin{aligned}
\chi_{r}= \begin{cases}-1, & \theta_{\text {single }}\left(t_{c_{4}},-1, \beta_{r}\right)=\theta_{\max } \\
1, & \text { else }\end{cases} \\
\chi_{l}= \begin{cases}-1, & \theta_{\text {single }}\left(t_{c_{2}}, 1, \beta_{l}\right)=\theta_{\max } \\
1, & \text { else }\end{cases}
\end{aligned}
$$

$$
\begin{aligned}
h_{\text {zone } 3}(t)= & 2 h\left(\theta\left(t,-1, t_{c_{6}}, \theta_{\text {max }}, t_{c_{5}}, \theta_{\text {min }}, \beta_{o}\right), \theta\left(t,-1, t_{c_{6}}, \theta_{\text {max }}, t_{c_{6}}, \theta_{\text {max }}, \beta_{o}\right)\right)+ \\
& h\left(\theta\left(t, \chi_{r}, t_{c_{4}}, \theta_{\text {max }}, t_{c_{3}}, \theta_{\text {min }}, \beta_{r}\right), \theta\left(t, \chi_{r}, t_{c_{4}}, \theta_{\text {max }}, t_{c_{4}}, \theta_{\text {max }}, \beta_{r}\right)\right)- \\
& h\left(\theta\left(t, \chi_{l}, t_{c_{2}}, \theta_{\text {max }}, t_{c_{1}}, \theta_{\text {min }}, \beta_{l}\right), \theta\left(t, \chi_{l}, t_{c_{2}}, \theta_{\text {max }}, t_{c_{2}}, \theta_{\text {max }}, \beta_{l}\right)\right),
\end{aligned}
$$


The determination of the sign for $\chi_{l, r}$ is needed because the nature of the angles at the side edges of the transducer is dependent on the point being before or after the elevation focus for a translated and rotated coordinate system to these edges. This means that if the location of the $\mathrm{z}$-coordinate of the point, $\mathrm{P}$, is before or after the elevation focus at the edge when the coordinates $x_{p}, y_{p}, z_{p}$ are transformed to a coordinate system located at the given edge, it alters the sign of $\chi_{r, l}$. A reformulation of the zone definitions may avoid this problem, but for the zone definitions applied in this work it is valid.

\section{Zone 4}

For Zone 4 the equations are dependent on $\gamma_{L} \leq \gamma_{p} \leq \gamma_{R}$. For $\gamma_{L} \leq \gamma_{p}$ the formulation becomes

$$
\begin{aligned}
h_{\text {zone4 }}(t)= & h\left(\theta\left(t,-1, t_{c_{2}}, \theta_{\text {max }}, t_{c_{L}}, \theta_{c_{L}}, \beta_{l}\right], \theta\left(t,-1, t_{c_{2}}, \theta_{\text {max }}, t_{c_{2}}, \theta_{\text {max }}, \beta_{l}\right)\right)+ \\
& h\left(\theta\left(t, 1, t_{c_{1}}, \theta_{\text {min }}, t_{c_{1}}, \theta_{\text {min }}, \beta_{l}\right), \theta\left(t, 1, t_{c_{1}}, \theta_{\text {min }}, t_{c_{L}}, \theta_{c_{L}}, \beta_{l}\right)\right)- \\
& h\left(\theta\left(t,-1, t_{c_{4}}, \theta_{\text {max }}, t_{c_{R}}, \theta_{c_{R}}, \beta_{r}\right), \theta\left(t,-1, t_{c_{4}}, \theta_{\text {max }}, t_{c_{4}}, \theta_{\text {max }}, \beta_{r}\right)\right)- \\
& h\left(\theta\left(t, 1, t_{c_{3}}, \theta_{\text {min }}, t_{c_{3}}, \theta_{\text {min }}, \beta_{r}\right), \theta\left(t, 1, t_{c_{3}}, \theta_{\text {min }}, t_{c_{R}}, \theta_{c_{R}}, \beta_{r}\right)\right),
\end{aligned}
$$

and for $\gamma_{p} \leq \gamma_{R}$ the formulation becomes

$$
\begin{aligned}
h_{\text {zone4 }}(t)= & h\left(\theta\left(t,-1, t_{c_{4}}, \theta_{\text {max }}, t_{c_{R}}, \theta_{c_{R}}, \beta_{r}\right), \theta\left(t,-1, t_{c_{4}}, \theta_{\text {max }}, t_{c_{4}}, \theta_{\text {max }}, \beta_{r}\right)\right)+ \\
& h\left(\theta\left(t, 1, t_{c_{3}}, \theta_{\text {min }}, t_{c_{3}}, \theta_{\text {min }}, \beta_{r}\right), \theta\left(t, 1, t_{c_{3}}, \theta_{\text {min }}, t_{c_{R}}, \theta_{c_{R}}, \beta_{r}\right)\right)- \\
& h\left(\theta\left(t,-1, t_{c_{2}}, \theta_{\text {max }}, t_{c_{L}}, \theta_{c_{L}}, \beta_{l}\right), \theta\left(t,-1, t_{c_{2}}, \theta_{\text {max }}, t_{c_{2}}, \theta_{\text {max }}, \beta_{l}\right)\right)- \\
& h\left(\theta\left(t, 1, t_{c_{1}}, \theta_{\text {min }}, t_{c_{1}}, \theta_{\text {min }}, \beta_{l}\right), \theta\left(t, 1, t_{c_{1}}, \theta_{\text {min }}, t_{c_{L}}, \theta_{c_{L}}, \beta_{l}\right)\right),
\end{aligned}
$$




\section{Zone 5}

For Zone 5 only one expression is needed for describing the SIR

$$
\begin{aligned}
h_{\text {zone } 5}(t)= & 2 h\left(\theta\left(t, 1, t_{c_{5}}, \theta_{\text {min }}, t_{c_{5}}, \theta_{\text {min }}, \beta_{0}\right), \theta\left(t, 1, t_{c_{5}}, \theta_{\text {min }}, t_{c_{0}}, \theta_{0}, \beta_{0}\right)\right)+ \\
& 2 h\left(\theta\left(t,-1, t_{c_{6}}, \theta_{\text {max }}, t_{c_{0}}, \theta_{0}, \beta_{0}\right), \theta\left(t,-1, t_{c_{6}}, \theta_{\text {max }}, t_{c_{6}}, \theta_{\text {max }}, \beta_{0}\right)\right)- \\
& h\left(\theta\left(t, 1, t_{c_{3}}, \theta_{\text {min }}, t_{c_{3}}, \theta_{\text {min }}, \beta_{r}\right), \theta\left(t, 1, t_{c_{3}}, \theta_{\text {min }}, t_{c_{R}}, \theta_{R}, \beta_{r}\right)\right)- \\
& h\left(\theta\left(t,-1, t_{c_{4}}, \theta_{\text {max }}, t_{c_{R}}, \theta_{R}, \beta_{r}\right), \theta\left(t,-1, t_{c_{4}}, \theta_{\text {max }}, t_{c_{4}}, \theta_{\text {max }}, \beta_{r}\right)\right)- \\
& h\left(\theta\left(t, 1, t_{c_{1}}, \theta_{\text {min }}, t_{c_{1}}, \theta_{\text {min }}, \beta_{l}\right), \theta\left(t, 1, t_{c_{1}}, \theta_{\text {min }}, t_{c_{L}}, \theta_{L}, \beta_{l}\right)\right)- \\
& h\left(\theta\left(t,-1, t_{c_{2}}, \theta_{\text {max }}, t_{c_{L}}, \theta_{L}, \beta_{l}\right), \theta\left(t,-1, t_{c_{2}}, \theta_{\text {max }}, t_{c_{2}}, \theta_{\text {max }}, \beta_{l}\right)\right) .
\end{aligned}
$$

\section{Zone 6}

In Zone 6 four different cases are defined. Zone 6 is located to the left and to the right of the transducer and the sign of $\chi$ is dependent on $y_{p}<0$ or $y_{p}>0$.

For $y_{p}<0$ and $\gamma_{p}<\gamma_{R}$ the SIRs are formulated as

$$
\begin{aligned}
h_{\text {zone6 }}(t)= & h\left(\theta\left(t, 1, t_{c_{4}}, \theta_{\text {max }}, t_{c_{3}}, \theta_{\text {min }}, \beta_{r}\right), \theta\left(t, 1, t_{c_{4}}, \theta_{\text {max }}, t_{c_{4}}, \theta_{\text {max }}, \beta_{r}\right)\right)- \\
& h\left(\theta\left(t, 1, t_{c_{2}}, \theta_{\text {max }}, t_{c_{1}}, \theta_{\text {min }}, \beta_{l}\right), \theta\left(t, 1, t_{c_{2}}, \theta_{\text {max }}, t_{c_{2}}, \theta_{\text {max }}, \beta_{l}\right)\right) .
\end{aligned}
$$

For $y_{p}>0$ and $\gamma_{p}<\gamma_{R}$ the SIR is formulated as

$$
\begin{aligned}
h_{\text {zone6 }}(t)= & h\left(\theta\left(t,-1, t_{c_{3}}, \theta_{\text {min }}, t_{c_{3}}, \theta_{\text {min }}, \beta_{r}\right), \theta\left(t,-1, t_{c_{3}}, \theta_{\text {min }}, t_{c_{4}}, \theta_{\text {max }}, \beta_{r}\right)\right)- \\
& h\left(\theta\left(t,-1, t_{c_{1}}, \theta_{\text {min }}, t_{c_{1}}, \theta_{\text {min }}, \beta_{l}\right), \theta\left(t,-1, t_{c_{1}}, \theta_{\text {min }}, t_{c_{2}}, \theta_{\text {max }}, \beta_{l}\right)\right) .
\end{aligned}
$$

For $y_{p}<0$ and $\gamma_{p}>\gamma_{R}$ the spatial impulse response is formulated as

$$
\begin{aligned}
h_{\text {zone6 }}(t)= & h\left(\theta\left(t, 1, t_{c_{2}}, \theta_{\text {max }}, t_{c_{1}}, \theta_{\text {min }}, \beta_{l}\right), \theta\left(t, 1, t_{c_{2}}, \theta_{\text {max }}, t_{c_{2}}, \theta_{\text {max }}, \beta_{l}\right)\right)- \\
& h\left(\theta\left(t, 1, t_{c_{4}}, \theta_{\text {max }}, t_{c_{3}}, \theta_{\text {min }}, \beta_{r}\right), \theta\left(t, 1, t_{c_{4}}, \theta_{\text {max }}, t_{c_{4}}, \theta_{\text {max }}, \beta_{r}\right)\right) .
\end{aligned}
$$

For $y_{p}>0$ and $\gamma_{p}>\gamma_{R}$ the spatial impulse response is formulated as

$$
\begin{aligned}
h_{\text {zone6 }}(t)= & h\left(\theta\left(t,-1, t_{c_{1}}, \theta_{\text {min }}, t_{c_{1}}, \theta_{\text {min }}, \beta_{l}\right), \theta\left(t,-1, t_{c_{1}}, \theta_{\text {min }}, t_{c_{2}}, \theta_{\text {max }}, \beta_{l}\right)\right)- \\
& h\left(\theta\left(t,-1, t_{c_{3}}, \theta_{\text {min }}, t_{c_{3}}, \theta_{\text {min }}, \beta_{r}\right), \theta\left(t,-1, t_{c_{3}}, \theta_{\text {min }}, t_{c_{4}}, \theta_{\text {max }}, \beta_{r}\right)\right) .
\end{aligned}
$$




\section{Zone 7}

This zone is dependent on the sign of $y_{p}$

For $y_{p}>0$ the SIR becomes

$$
\begin{aligned}
h_{\text {zone7 } 7}(t)= & 2 h\left(\theta\left(t,-1, t_{c_{0}}, \theta_{\text {min }}, t_{c_{0}}, \theta_{\text {min }}, \beta_{0}\right), \theta\left(t,-1, t_{c_{0}}, \theta_{\text {min }}, t_{c_{6}}, \theta_{\text {max }}, \beta_{0}\right)\right)- \\
& h\left(\theta\left(t,-1, t_{c_{3}}, \theta_{\text {min }}, t_{c_{3}}, \theta_{\text {min }}, \beta_{r}\right), \theta\left(t,-1, t_{c_{3}}, \theta_{\text {min }}, t_{c_{4}}, \theta_{\text {max }}, \beta_{r}\right)\right)- \\
& h\left(\theta\left(t,-1, t_{c_{1}}, \theta_{\text {min }}, t_{c_{1}}, \theta_{\text {min }}, \beta_{l}\right), \theta\left(t,-1, t_{c_{1}}, \theta_{\text {min }}, t_{c_{2}}, \theta_{\text {max }}, \beta_{l}\right)\right) .
\end{aligned}
$$

For $y_{p}<0$ the SIR becomes

$$
\begin{aligned}
h_{\text {zone } 7}(t)= & 2 h\left(\theta\left(t, 1, t_{c_{0}}, \theta_{\text {max }}, t_{c_{5}}, \theta_{\text {min }}, \beta_{0}\right), \theta\left(t, 1, t_{c_{0}}, \theta_{\text {max }}, t_{c_{0}}, \theta_{\text {max }}, \beta_{0}\right)\right)- \\
& h\left(\theta\left(t, 1, t_{c_{4}}, \theta_{\text {max }}, t_{c_{3}}, \theta_{\text {min }}, \beta_{r}\right), \theta\left(t, 1, t_{c_{4}}, \theta_{\text {max }}, t_{c_{4}}, \theta_{\text {max }}, \beta_{r}\right)\right)- \\
& h\left(\theta\left(t, 1, t_{c_{2}}, \theta_{\text {max }}, t_{c_{1}}, \theta_{\text {min }}, \beta_{l}\right), \theta\left(t, 1, t_{c_{2}}, \theta_{\text {max }}, t_{c_{2}}, \theta_{\text {max }}, \beta_{l}\right)\right) .
\end{aligned}
$$

\section{Zone 8}

Zone 8 is very similar to Zone 6 , where four conditions were found and it relies on finding the sign of $\chi$ as it was seen in Zone 3 .

For $y_{p}<0$ and $\gamma_{p}<\gamma_{R}$ the SIR is formulated as

$$
\begin{gathered}
\chi_{r}=\left\{\begin{array}{l}
-1, \theta_{\text {single }}\left(t_{c_{4}},-1, \beta_{r}\right)==\theta_{\text {max }} \\
1, \quad \text { else }
\end{array}\right. \\
\chi_{l}=\left\{\begin{array}{l}
-1, \theta_{\text {single }}\left(t_{c_{2}},-1, \beta_{l}\right)==\theta_{\text {max }} \\
1, \quad \text { else }
\end{array}\right. \\
h_{\text {zone8 }}(t)=h\left(\theta\left(t, \chi_{r}, t_{c_{4}}, \theta_{\text {max }}, t_{c_{3}}, \theta_{\text {min }}, \beta_{r}\right), \theta\left(t, \chi_{r}, t_{c_{4}}, \theta_{\text {max }}, t_{c_{4}}, \theta_{\text {max }}, \beta_{r}\right)\right)- \\
h\left(\theta\left(t, \chi_{l}, t_{c_{2}}, \theta_{\text {max }}, t_{c_{1}}, \theta_{\text {min }}, \beta_{l}\right), \theta\left(t, \chi_{l}, t_{c_{2}}, \theta_{\text {max }}, t_{c_{2}}, \theta_{\text {max }}, \beta_{l}\right)\right) .
\end{gathered}
$$

For $y_{p}>0$ and $\gamma_{p}<\gamma_{R}$ the SIR is formulated as 


$$
\begin{gathered}
\chi_{r}= \begin{cases}1, & \theta_{\text {single }}\left(t_{c_{3}}, 1, \beta_{r}\right)==\theta_{\text {min }} \\
-1, & \text { else }\end{cases} \\
\chi_{l}= \begin{cases}-1, & \theta_{\text {single }}\left(t_{c_{1}},-1, \beta_{l}\right)==\theta_{\text {min }} \\
1, & \text { else }\end{cases}
\end{gathered}
$$

$$
\begin{aligned}
h_{\text {zone8 } 8}(t)= & h\left(\theta\left(t, \chi_{r}, t_{c_{3}}, \theta_{\text {min }}, t_{c_{3}}, \theta_{\text {min }}, \beta_{r}\right), \theta\left(t, \chi_{r}, t_{c_{3}}, \theta_{\text {min }}, t_{c_{4}}, \theta_{\text {max }}, \beta_{r}\right)\right)- \\
& h\left(\theta\left(t, \chi_{l}, t_{c_{1}}, \theta_{\text {min }}, t_{c_{1}}, \theta_{\text {min }}, \beta_{l}\right), \theta\left(t, \chi_{l}, t_{c_{1}}, \theta_{\text {min }}, t_{c_{2}}, \theta_{\text {max }}, \beta_{l}\right)\right) .
\end{aligned}
$$

For $y_{p}>0$ and $\gamma_{p}>\gamma_{L}$ the SIR is formulated as

$$
\begin{gathered}
\chi_{l}=\left\{\begin{array}{l}
1, \quad \theta_{\text {single }}\left(t_{c_{1}}, 1, \beta_{l}\right)=\theta_{\text {min }} \\
-1, \text { else }
\end{array}\right. \\
\chi_{r}= \begin{cases}1, & \theta_{\text {single }}\left(t_{c_{3}}, 1, \beta_{r}\right)==\theta_{\text {max }} \\
-1, & \text { else }\end{cases} \\
h_{\text {zone } 8}(t)=h\left(\theta\left(t, \chi_{l}, t_{c_{1}}, \theta_{\text {min }}, t_{c_{1}}, \theta_{\text {min }}, \beta_{l}\right), \theta\left(t, \chi_{l}, t_{c_{1}}, \theta_{\text {min }}, t_{c_{2}}, \theta_{\text {max }}, \beta_{l}\right)\right)- \\
h\left(\theta\left(t, \chi_{r}, t_{c_{3}}, \theta_{\text {min }}, t_{c_{3}}, \theta_{\text {min }}, \beta_{r}\right), \theta\left(t, \chi_{r}, t_{c_{3}}, \theta_{\text {min }}, t_{c_{4}}, \theta_{\text {max }}, \beta_{r}\right)\right) .
\end{gathered}
$$




\section{Zone 9}

This zone is located at the elevation focus of the transducer. Considering (8) and setting $R=\left|O P^{*}\right|$ and $y_{p}=0$ yields

$$
h_{f}\left(t_{i}\right)=\frac{1}{2 \pi} \int_{\theta_{\min }\left(t_{i}\right)}^{\theta_{\max }\left(t_{i}\right)} \frac{c r}{R \sqrt{1-\left(1+\frac{r^{2}-c^{2} t_{i}^{2}}{2 R^{2}-2 r R \sin \theta}\right)^{2}}} d \theta .
$$

For the initial time step, where $r^{2}-c^{2} t_{i}^{2}=0$, the integral is infinite, which is both a numerical problem and physically not appropriate. This has to be accounted for in a given implementation by either finding an asymptotic value using the gradient of the integration values from time $t>t_{\text {minimum }}$ and the following time steps, or by finding the initial step from a point located just before the elevation focus as well as one located just after the elevation focus. These initial values may give an approximate mean value of the initial samples at the elevation focus. The mean energy between these two samples may then be distributed across the samples.

The SIR takes on the following form

$$
\begin{aligned}
h_{\text {zone9 } 9}(t)= & 2 h_{f}\left(\theta\left(t, 1, t_{c_{0}}, \theta_{\text {min }}, t_{c_{0}}, \theta_{\text {min }}, \beta_{0}\right), \theta\left(t, 1, t_{c_{0}}, \theta_{\text {max }}, t_{c_{0}}, \theta_{\max }, \beta_{0}\right)\right)-\ldots \\
& h_{f}\left(\theta\left(t, 1, t_{c_{L}}, \theta_{L}, t_{c_{1}}, \theta_{\text {min }}, \beta_{l}\right), \theta\left(t,-1, t_{c_{L}}, \theta_{L}, t_{c_{2}}, \theta_{\max }, \beta_{l}\right)\right)-\ldots \\
& h_{f}\left(\theta\left(t, 1, t_{c_{R}}, \theta_{R}, t_{c_{3}}, \theta_{\text {min }}, \beta_{r}\right), \theta\left(t,-1, t_{c_{R}}, \theta_{R}, t_{c_{4}}, \theta_{\max }, \beta_{r}\right)\right) .
\end{aligned}
$$

Notice that $\theta\left(t, 1, t_{c_{0}}, \theta_{\min }, t_{c_{0}}, \theta_{\min }, \beta_{0}\right)$ for the first integration equals $\theta_{\min }$ and $\theta\left(t, 1, t_{c_{0}}, \theta_{\max }, t_{c_{0}}, \theta_{\max }, \beta_{0}\right)$ equals $\theta_{\max }$ for all time instants.

\section{Zone 10}

This zone is similar to Zone 2 and only differs at $y_{p}=0$. All time of flight calculations are the same. Also the SIR is given by (25) and (26) as for Zone 2. 


\section{APPROXIMATING THE INTEGRAL EXPRESSION}

The integral in (8) is of elliptical type and has no direct analytical solution. However, the following analysis of the integral will show that an approximation of the integrand makes the SIR analytically integrable.

Consider (8) in the following form

$$
h=\frac{1}{2 \pi} \int_{\phi_{\min }}^{\phi_{\max }} \frac{c r}{\left|O P^{*}\right| \sqrt{1-P(\theta)}} d \theta,
$$

where

$$
P(\theta)=\frac{\left(-k+c^{2} t^{2}+2 r y_{p} \cos \theta+2 r R \sin \theta\right)^{2}}{\left(2\left|O P^{*}\right| R-2\left|O P^{*}\right| r \sin \theta\right)^{2}} .
$$

Example plots for the integrand at several time instant are seen in Fig. 6. The vertical lines indicate the location of the $\phi_{\min }$ and $\phi_{\max }$ at different time steps and as indicated by the form of (57) the integrand approaches infinity for $P(\theta) \rightarrow 1$.

Because of the integrand's nature it gets difficult, (but not impossible), to perform a series expansion that can replace the integrand and reveal an analytically integrable integrand without introducing a significant error in energy conservation close to the maximum integration angles. As a consequence this method may not be the most effective one to apply.

It may be more beneficial to consider the expression in (57) and apply a second order Taylor expansion to this polynomial and achieve a second order polynomial, $T\left(\theta, \theta_{0}\right)$, around a local integration angle $\theta_{0}$.

$$
T\left(\theta, \theta_{0}\right)=A\left(\theta_{0}\right) \theta^{2}+B\left(\theta_{0}\right) \theta+C\left(\theta_{0}\right)
$$

where $A\left(\theta_{0}\right), B\left(\theta_{0}\right)$, and $C\left(\theta_{0}\right)$ are the coefficients found by ordering the Taylor series.

Expanding the polynomial into only a second order is beneficial in this work, contrary higher order expansions, since the indefinite integral of the SIR becomes analytically inte- 


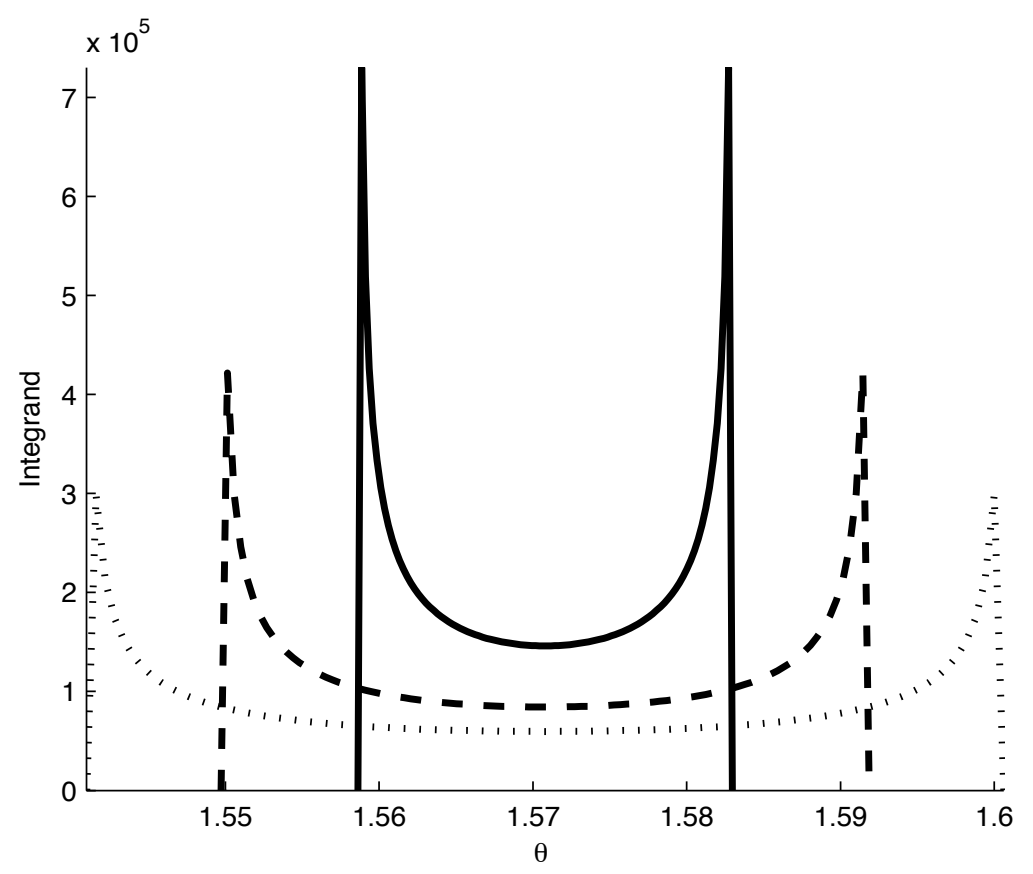

FIG. 6. A curve shape of the integrand in (56) at different time steps. It is clearly seen how the curve increases asymptotic forward infinity at $\phi_{\min }$ and $\phi_{\max }$. Notice that a full symmetric case is shown for the plot. Symmetry is always the case, however, depending on the value of $\phi_{\min }$ and $\phi_{\max }$ one or both spikes at the start and end of the integration domain may not be present.

grable

$$
\begin{aligned}
\text { Int } & =\frac{1}{2 \pi} \int \frac{c r}{\left|O P^{*}\right| \sqrt{1-\left(A \theta^{2}+B \theta+C\right)}} d \theta \\
& =\frac{1}{2 \pi} \frac{c r \tan ^{-1}\left[\frac{B+2 A \theta}{2 \sqrt{A} \sqrt{1-C-B \theta-A \theta^{2}}}\right]}{\left|O P^{*}\right| \sqrt{A}} \\
& =\frac{1}{2 \pi} \frac{i c r \log \left[2 \sqrt{-A \theta^{2}-B \theta-C+1}-\frac{i(2 A \theta+B)}{\sqrt{A}}\right]}{\sqrt{A}\left|O P^{*}\right|},
\end{aligned}
$$

where $i=\sqrt{-1}$.

An integration of (56) can be found by performing the second order Taylor expansion around a sufficient number of $\theta_{0}$ angles within the interval of $\phi_{\min }$ and $\phi_{\max }$ and then one makes the corresponding sub integrations. This will, however, compromise the desired benefit of a fast computational expression in the analytical expression. 

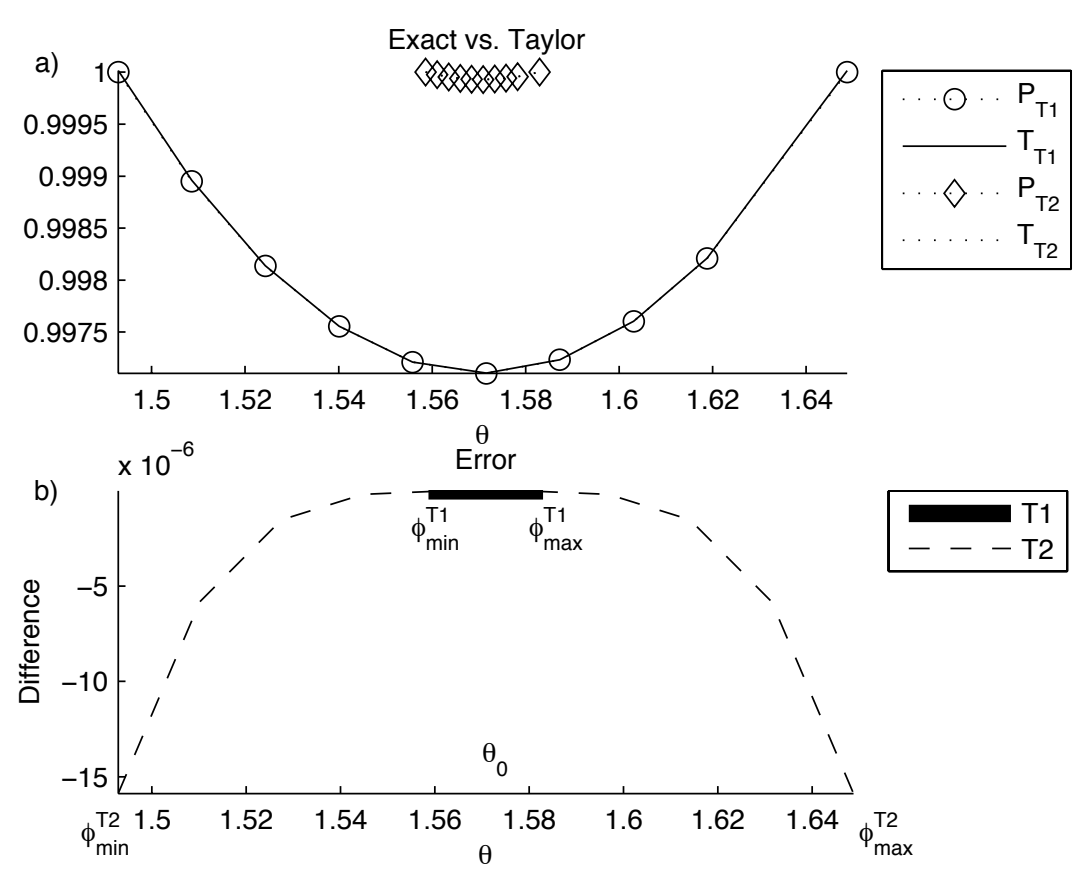

FIG. 7. a) A comparison between $P(\theta)$ and $T(\theta)$ at two time instants, $\mathrm{T} 1$ and $\mathrm{T} 2$, where $\mathrm{T} 1<\mathrm{T} 2$. b) The difference between $P(\theta)$ and $T(\theta)$ at the two time instants. Notice how $\phi_{\min }$ and $\phi_{\max }$ include a wider angle difference for $T 2$ and how the error has increased significantly.

Consider Fig. 7a. This figure shows how the second order polynomial, $T\left(\theta, \theta_{0}\right)$, fits (57) for $\theta_{0}=\frac{\phi_{\min }+\phi_{\max }}{2}$ at different time steps. It is seen that when a small angle interval $\Delta \phi=\phi_{\max }-\phi_{\min }$ is considered, as for the $\mathrm{T}_{1}$ example, a very close curve fit is possible. However, Fig. $7 \mathrm{~b}$. shows that for bigger $\Delta \phi$, as for the $\mathrm{T}_{2}$ example, the residual of the expansion becomes of more and more influence at the outer integration boundaries, which is a natural consequence of the Taylor expansion. This is an undesirable consequence that becomes very important for calculations on large transducers, since a significant amount of energy is located in the neighborhood of $\phi_{\min }$ and $\phi_{\max }$ as shown in Fig. 6.

A strategy for capturing the energy at the outer integration boundaries could therefore be to perform Taylor expansions at $\phi_{\min }, \phi_{\max }$, and $\frac{\phi_{\min }+\phi_{\max }}{2}$ and then subdivide the 
integration into three intervals:

$$
\left(\phi_{\min } \rightarrow \frac{\phi_{\min }+\phi_{0}}{2}\right), \quad\left(\frac{\phi_{\min }+\phi_{0}}{2} \rightarrow \frac{\phi_{\max }+\phi_{0}}{2}\right), \quad\left(\frac{\phi_{\max }+\phi_{0}}{2} \rightarrow \phi_{\max }\right)
$$

This method ensures better conservation of the energy near the outer integration angles than a single expansion around the mean integration value does. Furthermore, it captures the centered curvature. The cost of this method is however three times more calculation time for small angles.

\section{SIMULATIONS}

To test the developed algorithms an adaptive Gauss-Kronrod quadrature numerical integrator from MATLAB is applied for solving the exact form of the integral in (8). As reference to validate the pulse shape of the exact solution a high resolution transducer model in Field II is used. Furthermore, to approximate the exact integral of the SIR simulations with a three-point Taylor expansion, a single-point Taylor expansion at the mean integration angle, and a direct second order polynomial fit are used. The latter implementation uses three points to find the coefficients of a second order polynomial. These three points are $P\left[\phi_{\min }\right]$, $P\left[\left(\phi_{\min }+\phi_{\max }\right) / 2\right]$, and $P\left[\phi_{\max }\right]$. The latter method differs from the three-point Taylor approximation, because it finds a best polynomial fit through the three points and not a local fit as the Taylor expansion does.

The error is calculated as

$$
E_{i}=100 \frac{\sqrt{\frac{1}{N} \sum_{N}\left(h_{i}-h_{\text {numerical }}\right)^{2}}}{\sqrt{\frac{1}{N} \sum_{N} h_{\text {numerical }}^{2}}},
$$

where $h_{\text {numerical }}$ is the exact solution to the SIR as represented by (8) and solved using the numerical integrator. $h_{i}$ is the SIR calculated with either Field II, $h_{F}$, the three-point Taylor expansion, $h_{3 T}$, the single-point Taylor expansion, $h_{1 T}$, or the second order polynomial fit, $h_{2 p} . N$ is the number of samples in the response. To get sample times aligned a simple spline interpolation between the points are performed. This is necessary since start times for the 
pulses may be calculated slightly differently in Field II than in the MATLAB implementation applied in this work.

A double curved transducer with a width of $20 \mathrm{~mm}$ and a $30 \mathrm{~mm}$ height is simulated. The outer radius, $\mathrm{R}$, is $60 \mathrm{~mm}$ and the inner radius, $r$, is $90 \mathrm{~mm}$. Initially a single response from a point located in Zone 1 at $\left\{x_{p}, y_{p}, z_{p}\right\}=\{0,0,10\} \mathrm{mm}$ is calculated. The coordinates are defined using the coordinate definition of Field II. The zero reference for the $\mathrm{z}$-coordinate is located at the outer most z-coordinates for the transducer defined in the torus coordinates. The sampling frequency is set to $5 \mathrm{GHz}$. This relatively high sampling frequency is used to avoid energy loss due to numerically difficult cases at edges and focus points. A comprehensive discussion on this can be found in the discussion section. A second simulation case investigates 200 points randomly distributed in front of the transducer and covering all zones.

A third simulation investigates the error for the situation where $R=90 \mathrm{~mm}$ and $r=6$ m by comparing $h_{3 T}$ and $h_{1 T}$ with Field II as reference. The mean error is calculated for 200 points randomly distributed across the zones. This simulation will due to the transducers' large inner radius, $r$, mimic a convex transducer with no elevation focus. Yet a fourth simulation investigates $R=6 \mathrm{~m}$ and $r=6 \mathrm{~m}$ which corresponds to a plane transducer and the mean error of simulating 200 points is calculated. Finally a study case where $R=6 \mathrm{~m}$ and $r=60 \mathrm{~mm}$ is performed. This type of transducer corresponds to a elevation focused linear rectangular transducer. The transducer dimensions for simulation case three, four, and five are changed to a more realistic size with a width of $1 \mathrm{~mm}$ and a height of $10 \mathrm{~mm}$. The sampling frequency is fixed at $5 \mathrm{GHz}$.

\section{RESULTS}

Figure $8 \mathrm{a}$ shows the results of simulating the double curved transducer, i.e. $R=60$ $\mathrm{mm}$ and $r=90 \mathrm{~mm}$ at the point $\left\{x_{p}, y_{p}, z_{p}\right\}=\{0,0,10\} \mathrm{mm}$. Clearly all the solvers agree visually on the result from a full pulse perspective and a zoom as shown in Fig. 8b is needed 

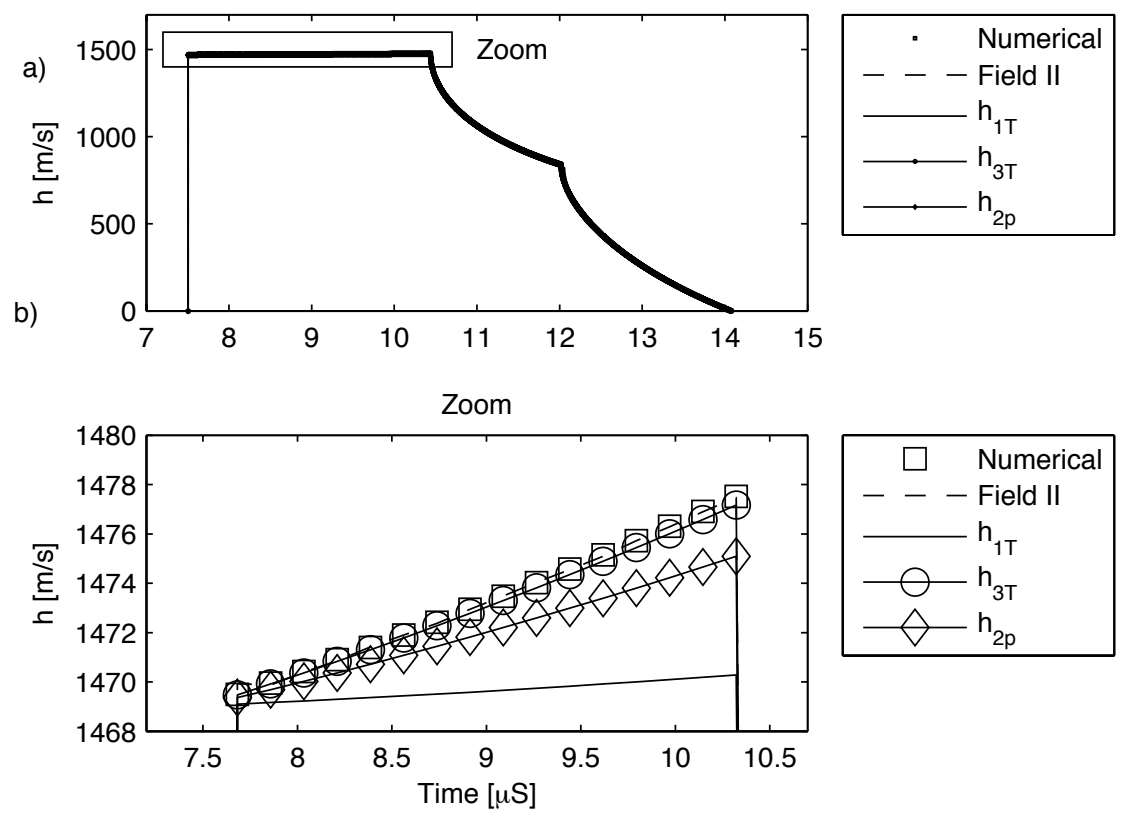

FIG. 8. Results of simulating a single point in front of a double curved transducer. a) Full pulse profile. b) Zoom onto (a) to magnify the difference.

to visually identify the difference. The relative errors were found to be: $E_{\text {Field }}=0.40 \%$, $E_{1 T}=0.80 \%, E_{3 T}=0.03 \%$, and $E_{2 p}=0.18 \%$. A higher exactness for the $3 T$ approximator was seen compared to the other solvers. Performing the same simulation for 200 points randomly distributed across all zones resulted in a mean error (ME) of: $\mathrm{ME}_{\text {Field }}=0.45 \%$, $\mathrm{ME}_{1 T}=1.78 \%, \mathrm{ME}_{3 T}=0.01 \%$, and $\mathrm{ME}_{2 p}=0.45 \%$. Also calculating the mean of the solving time, $\mathrm{T}_{i}$, for each solver yielded: $\mathrm{T}_{\text {Numerical }}=73.0 \mathrm{~s}, \mathrm{~T}_{1 T}=8.7 \mathrm{~s}, \mathrm{~T}_{3 T}=9.4 \mathrm{~s}$, and $\mathrm{T}_{2 p}=7.0 \mathrm{~s}$. Clearly the numerical solver is by far the slowest, which was also expected, however, the mean times show that an improvement in the error from $1.78 \%$ to $0.01 \%$ can be achieved with a $8.1 \%$ increase in simulation time by applying the three point approximator instead of the fast one point approximator or an improvement in the error from $0.45 \%$ to $0.01 \%$ with a $34.3 \%$ increase in simulation time by changing the solver from the polynomial fitting to the three point expansion.

Figure 9a shows a simulation for a point at $\left\{x_{p}, y_{p}, z_{p}\right\}=\{0,0,105\} \mathrm{mm}$. A point at this location introduces a sharp spike into the response. From Fig. 9a a zoom onto the spike in 


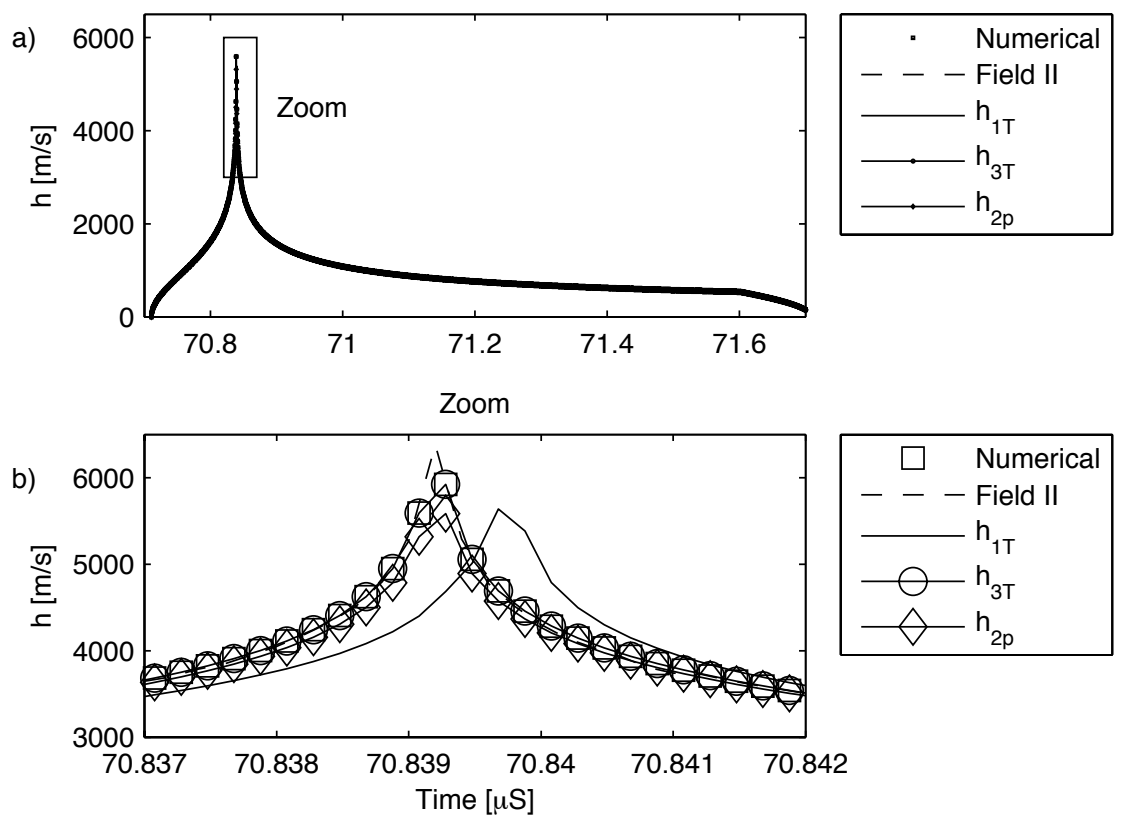

FIG. 9. Results of simulating a sharp spiking spatial impulse response from a double curved transducer. $E_{\text {Field }}=0.49 \%, E_{1 T}=3.580 \%, E_{3 T}=0.006 \%, E_{2 p}=0.83 \%$. Only a few data points are shown from each curve a) Full pulse profile. b) Zoom onto (a) to magnify the difference.

Fig. 9a is to be found. Clearly the one point solver $h_{1 T}$ is calculating the spike incorrectly. This may look like a wrong edge calculation for this solver, however, the implementation of edge calculation, zones etc. are identical with all the other zones, which are seen to calculate the response more correctly. The errors were found to be $E_{\text {Field }}=0.49 \%, E_{1 T}=3.58 \%$, $E_{3 T}=0.006 \%, E_{2 p}=0.83 \%$.

Figure 10a shows the result of simulating a point at $\left\{x_{p}, y_{p}, z_{p}\right\}=\{0,0,40\} \mathrm{mm}$ on a convex non-elevation focused transducer using the model presented in this work. Figure 10b shows a zoom from Fig. 10a of the horizontal line section. From the latter it can be seen that the $h_{1 T}$ curve is having difficulties in capturing the pulse shape. The error, $E_{i}$, relative to the numerical solution is $E_{\text {Field }}=6.9 \%, E_{1 T}=5.9 \%, E_{3 T}=0.01 \%, E_{2 p}=0.0283 \%$. This shows that the $3 \mathrm{~T}$ and the $2 \mathrm{p}$ are good solvers for convex arrays, and the error $E_{\text {Field }}$ shows that the algorithm has consistency with what Field II predicts. Further experiments 

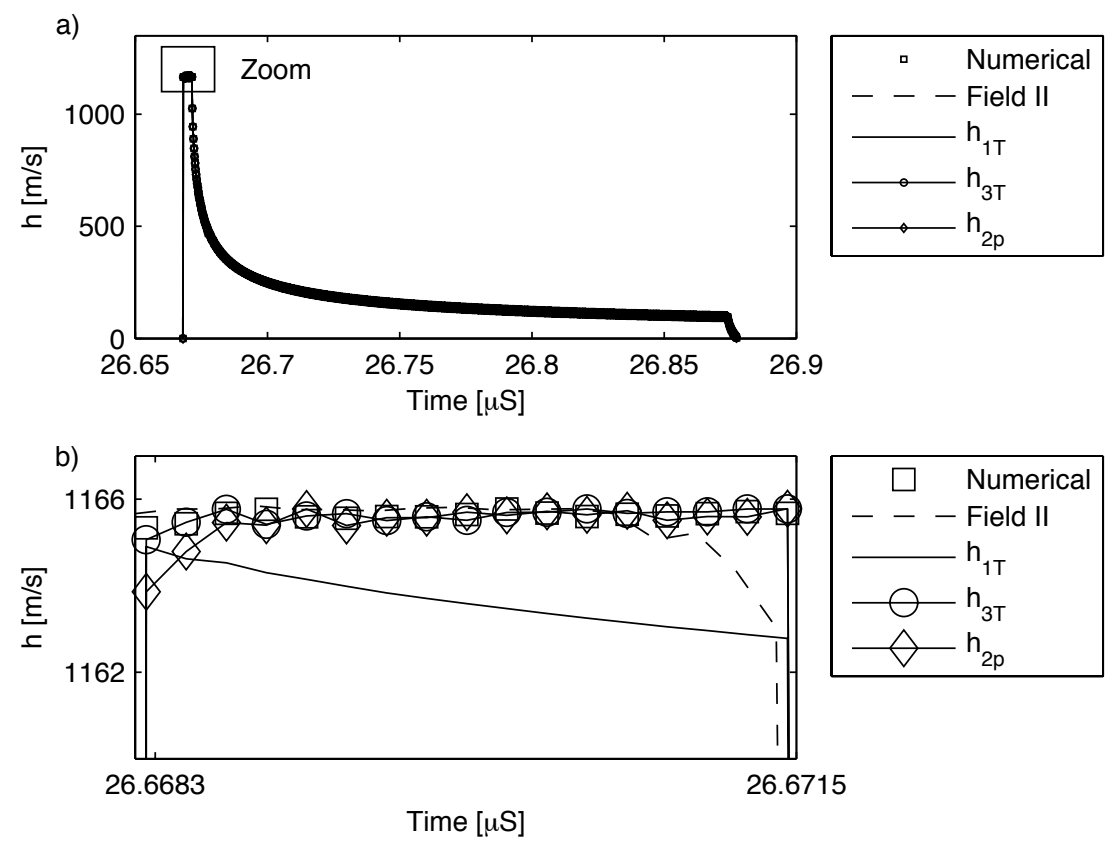

FIG. 10. Results of simulating a convex non-elevation focused array.

with simulations at points close to the convex transducer e.g. $\left\{x_{p}, y_{p}, z_{p}\right\}=\{0,0,1\} \mathrm{mm}$ have shown that the numerical integrator breaks down. However, the approximating models are still stable. Field II is therefore applied as the reference to get a measure of the error for simulations in all zones of a convex transducer. 200 points were investigated and the mean error for each solver was found to be $\mathrm{ME}_{1 T}=3.8 \%, \mathrm{ME}_{3 T}=2.5 \%, \mathrm{ME}_{2 p}=2.5 \%$, which indicate a rather identical performance of the solvers.

Changing the transducer geometry to a linear flat transducer $R=6 \mathrm{~m}$ and $r=6$ $\mathrm{m}$ for the model presented here and for Field II yields a mean error of $\mathrm{ME}_{1 T}=3.56 \%$, $\mathrm{ME}_{3 T}=3.56 \%, \mathrm{ME}_{2 p}=34.86 \%$ for 200 points and with Field II as the reference. The errors show that the model can capture the response from plane transducers as well. It also indicates that the polynomial fitting, $2 p$, fails to predict the response in contradiction to the $1 T$ and the $3 T$ analytical solvers.

Finally, a linear elevation focused array element was considered. This array element is mimicked by, $R=6 \mathrm{~m}, r=60 \mathrm{~mm}$, height $=10 \mathrm{~mm}$, and $\gamma_{L}=83 \mu \mathrm{rad}$, which corresponds to a width of approximately $1 \mathrm{~mm}$. The mean error relative to Field II was found to be 
$\mathrm{ME}_{1 T}=4.43 \%, \mathrm{ME}_{3 T}=3.46 \%$, and $\mathrm{ME}_{2 p}=33.30 \%$ for a simulation with 200 points. The mean solving time for the three approximating methods was found to be $\mathrm{T}_{1 T}=0.49 \mathrm{~s}$, $\mathrm{T}_{3 T}=0.57 \mathrm{~s}$, and $\mathrm{T}_{2 p}=0.33 \mathrm{~s}$. This implies an error improvement of $21.9 \%$ with a $15.4 \%$ increase in the simulation time when using the $3 \mathrm{~T}$ instead of the $1 \mathrm{~T}$ and a $89 \%$ improvement in the error when applying the $3 \mathrm{~T}$ instead of the $2 \mathrm{p}$ solver. The latter improvement costs $72.7 \%$ more calculation time.

\section{DISCUSSION}

The results have shown that accurate predictions of the exact solution to (8) could be achieved by using the three-point Taylor expansion, 3T, for all transducer configurations. However, also good results were achieved by calculating the second order polynomial and the one point Taylor expansion for the double curved transducer. As could be seen in Fig. 9 the $1 \mathrm{~T}$ calculations fails for steep spikes. This is because the main energy that represent the spike is found at the outer integration values $\phi_{\min }$ and $\phi_{\max }$. The $1 \mathrm{~T}$ solver in contrary seems to be more stable for linear arrays which the $2 \mathrm{p}$ showed not to be. This shows that the solvers $1 \mathrm{~T}$ and $2 \mathrm{p}$ are sensitive to the curvatures of the transducer. Choosing which solver that operates the best is therefore application dependent. Clearly the $3 \mathrm{~T}$ exhibits the best performance, but for the cost of a slight increase in the solution time relative to the other solvers. It should also be mentioned that the influence of miscalculating a spike as seen in Fig. 9 is significant when the pure shapes of the SIRs are to be compared. However, it should be recalled that the SIR are typically convolved with a band-limited pulse representing the transducer in emission or in pulse-echo. Therefore, when the SIR is used in a convolution an error, as the ones typical for the 1T, becomes of less influence. If the convolved pulse is sufficiently band-limited for the given application, it may be beneficial to calculate the responses with the slightly faster approximation of $1 \mathrm{~T}$ and $2 \mathrm{p}$. It should, however, be noted that the $8.1 \%$ percent increase in simulation time that the 3T approximation offers relatively to the $1 \mathrm{~T}$ or $34.3 \%$ relatively to the $2 \mathrm{p}$ is relatively small compared to the high accuracy 
and stability it represents for the double curved transducer.

From the results it is also noticeable that the algorithm is a fairly good approximation as a model for linear arrays, elevation focused linear arrays, and convex arrays with no elevation focus. The model therefore represents an all-round formulation of the SIR of general rectangular arrays. Further development should therefore focus on a fast and competitive implementation into a $\mathrm{C} /$ Fortran environment from which a simulation time comparison between recognized programs such as Ultrasim, Dream, DELFI, and Field II could be made. An initial C-implementation of the algorithms has been compared to Field II in the authors' conference contribution ${ }^{26}$. This latest contribution also considers a narrower double curved array element with a width and height of $250 \mu \mathrm{m}$ and $10 \mathrm{~mm}$, respectively.

Yet another aspect that should be discussed in the context of a practical implementation is the sampling frequency. The sampling frequency applied in this work is relatively large, 5 $\mathrm{GHz}$, which was chosen to validate the algorithm directly. A much lower sampling frequency should be applied if the algorithm and the solver should be implemented into a simulation program such as Field II, which is usually operated at a $100 \mathrm{MHz}$ and with small elements. The combination between small elements and low sampling frequency is only possible if the implementation preserves conservation of energy in the SIR. This may be achieved by performing an area integration of the pulse within the samples. This area integration is then spread out onto the different global samples. In other words a sub-integration procedure is to be performed and is a trivial task to perform.

\section{CONCLUSION}

An exact mathematical formulation for the SIR of a convex rectangular elevation focused transducer (double curved transducer) has been presented. The response can be represented with an integral of elliptical type. For this integral to be solved it requires a numerical integrator. It has been shown that a good approximation of the integral can be achieved by applying a three-point Taylor expansion to a part of the integral. The Taylor expansion 
yields an analytically integrable expression. A single-point Taylor expansion and a second order polynomial fit was also shown to give good results for simulations of a double curved transducer. The presented algorithm exhibited consistent results with Field II for a double curved transducer, a linear flat rectangular transducer, a linear elevation focused rectangular transducer, and a convex non-elevation focused rectangular transducer. The three-point Taylor approximation showed to be the most stable approximation, at the cost of a slightly higher simulation time.

\section{References}

1 G. E. Tupholme, "Generation of acoustic pulses by baffled plane pistons", Mathematika 16, 209-224 (1969).

2 P. R. Stepanishen, "Transient radiation from pistons in an infinite planar baffle", J. Acoust. Soc. Am. 49, 1629-1638 (1971).

3 P. R. Stepanishen, "The time-dependent force and radiation impedance on a piston in a rigid infinite planar baffle", J. Acoust. Soc. Am. 49, 841-849 (1971).

${ }^{4}$ P. R. Stepanishen, "An approach to compute time-dependent interaction forces and mutual radiation impedances between pistons in a rigid planar baffle", J. Acoust. Soc. Am. 49, 283-292 (1971).

5 J. C. Lockwood and J. G. Willette, "High-speed method for computing the exact solution for the pressure variations in the nearfield of a baffled piston", J. Acoust. Soc. Am. 53, 735-741 (1973).

${ }^{6}$ P. R. Stepanishen, "Wide bandwidth near and far field transients from baffled pistons", in Proc. IEEE Ultrason. Symp., 113-118 (1977).

7 G. R. Harris, "Review of transient field theory for a baffled planar piston", J. Acoust. Soc. Am. 70-1, 10-20 (1981).

8 J. A. Jensen, "A new calculation procedure for spatial impulse responses in ultrasound", J. Acoust. Soc. Am. 105, 3266-3274 (1999). 
9 C.-C. Sung, H.-I. Yang, and J.-H. Jeng, "Calculation of spatial impulse response for array transducer", Jap. J. Appl. Phys. 44-10, 7680-7689 (2005).

10 A. Penttinen and M. Luukkala, "The impulse response and nearfield of a curved ultrasonic radiator", J. Phys. D: Appl. Phys. 9, 1547-1557 (1976).

11 M. Arditi, F. S. Forster, and J. Hunt, "Transient fields of concave annular arrays", Ultrason. Imaging 3, 37-61 (1981).

12 J. N. Tjøtta and S-Tjøtta, "Nearfield and farfield of pulsed radiators", J. Acoust. Soc. Am. 71-4, 824-834 (1982).

13 W. A. Verhoef, M. J. T. M. Cloostermans, and J. M. Thijssen, "The impulse response of a focused source with an arbitrary axisymmetric surface velocity distribution", J. Acoust. Soc. Am. 75-6, 1716-1721 (1984).

14 J. A. Jensen, "Field: A program for simulating ultrasound systems", Med. Biol. Eng. Comp. 10th Nordic-Baltic Conference on Biomedical Imaging, Vol. 4, Supplement 1, Part 1, 351-353 (1996b).

15 J. A. Jensen, "Users' guide for the Field II program, version 2.70 of may 26, 1999", Technical Report, Department of Information Technology, DTU (1999).

16 J. A. Jensen and N. B. Svendsen, "Calculation of pressure fields from arbitrarily shaped, apodized, and excited ultrasound transducers", IEEE Trans. Ultrason., Ferroelec., Freq. Contr. 39, 262-267 (1992).

17 J. A. Jensen, "Ultrasound fields from triangular apertures", J. Acoust. Soc. Am. 100(4), 2049-2056 (1996a).

18 R. Reibold and R. Kažys, "Radiation of a rectangular strip-like focussing transducer. part1: harmonic excitation.", Ultrasonics 30, 49-55 (1992).

19 R. Reibold and R. Kažys, "Radiation of a rectangular strip-like focussing transducer. part2: transient excitation.", Ultrasonics 30, 49-55 (1992).

20 P. Wu and T. Stepinski, "Spatial impulse response method for predicting pulse-echo fields from a linear array with cylindrically concave surface", IEEE Trans. Ultrason., Ferroelec., Freq. Contr. 46-5, 1283-1297 (1999). 
21 J. F. Theumann, M. Arditi, J.-J. Meister, and E. Jaques, "Acoustic fields of concave cylindrical transducers", J. Acoust. Soc. Am. 88-2, 1160-1169 (1990).

22 S. Holm, "Ultrasim - a toolbox for ultrasound field simulation", University of Oslo (2001).

23 F. Lingvall, "User manual for the DREAM toolbox- an ultrasound simulation software for use with MATLAB and GNU OCTAVE", (2009).

24 M. A. Ellis, D. Guenther, and W. F. Walker, "Spline-based approach for computing spatial impulse responses", IEEE Trans. Ultrason., Ferroelec., Freq. Contr. 54-5, 10451054 (2007).

25 H. T. O'Neil, "Theory of focusing radiators", J. Acoust. Soc. Am. 21-5, 516-526 (1949).

26 D. Bæk, and J. A. Jensen, and M. Willatzen, "Testing of a spatial impulse response algorithm for double curved transducers",1226-1229 , Proc. IEEE Ultrason. Symp. (2010). 


\section{List of Figures}

FIG. 1 The geometrical definition of the double curved transducer. . . . . . . . 5

FIG. 2 Figure showing the geometrical definition of the angle $\beta \ldots \ldots 6$

FIG. 3 The definition of the integration angles $\theta_{\min }^{L}, \theta_{\text {max }}^{L}, \theta_{\text {min }}^{R}$, and $\theta_{\text {max }}^{R}$, which occur when the spherical wave has passed the boundaries of the transducer. The transducer is seen from the back side toward the positive z-axis. . . . .

FIG. 4 A sphere's crossing with a transducer (solid) and virtual crossings (dotted). View seen from the transducer's back side in direction of the z-axis. a) The sphere has not yet crossed the side edges. The sphere is symmetrically placed at $x_{p}=0$. b) The sphere has crossed the edges. Dotted lines indicate the virtual arc crossings. The sphere center is offset to the left, which makes the left virtual arc significantly longer than the right virtual arc. See AngleVideo. Link to AngleVideo.avi. . . . . . . . . . . . . . . . . . . .

FIG. 5 The integration angles plotted for a point located in Zone 1 of a transducer. The angles show that the wave crosses the left edge before it crosses the right. Start and end times are $t_{c_{0}}$ and $t_{c_{3}}$, respectively. . . . . . . . . . . . . 14

FIG. 6 A curve shape of the integrand in (56) at different time steps. It is clearly seen how the curve increases asymptotic forward infinity at $\phi_{\min }$ and $\phi_{\max }$. Notice that a full symmetric case is shown for the plot. Symmetry is always the case, however, depending on the value of $\phi_{\min }$ and $\phi_{\max }$ one or both spikes at the start and end of the integration domain may not be present.

FIG. 7 a) A comparison between $P(\theta)$ and $T(\theta)$ at two time instants, T1 and T2, where $\mathrm{T} 1<\mathrm{T} 2$. b) The difference between $P(\theta)$ and $T(\theta)$ at the two time instants. Notice how $\phi_{\min }$ and $\phi_{\max }$ include a wider angle difference for $T 2$ and how the error has increased significantly. . . . . . . . . . .

FIG. 8 Results of simulating a single point in front of a double curved transducer.

a) Full pulse profile. b) Zoom onto (a) to magnify the difference. . . . . . . 26 
FIG. 9 Results of simulating a sharp spiking spatial impulse response from a double curved transducer. $E_{\text {Field }}=0.49 \%, E_{1 T}=3.580 \%, E_{3 T}=0.006 \%, E_{2 p}=$ $0.83 \%$. Only a few data points are shown from each curve a) Full pulse profile. b) Zoom onto (a) to magnify the difference. . . . . . . . . . . . . . . 27

FIG. 10 Results of simulating a convex non-elevation focused array. . . . . . . . . . 28 